\title{
Faster blood flow rate does not improve circuit life in Continuous Renal Replacement
}

\section{Therapy: A randomized controlled trial}

Nigel Fealy RN, $\mathrm{MN}^{1,2,3}$, Leanne Aitken RN, PhD, FACCCN $2,4,5,6$, Eugene du Toit $\mathrm{PhD}^{7}$, Serigne Lo PhD, AStat ${ }^{8}$, lan Baldwin RN, PhD, FACCCN ${ }^{1,3}$

1. Department of Intensive Care Medicine, Austin Hospital, Melbourne, Australia

2. School of Nursing and Midwifery, Griffith University, Brisbane, Australia

3. School of Nursing and Midwifery, Deakin University, Melbourne, Australia

4. Centre for Health Practice Innovation, Griffith Health Institute, Griffith University, Brisbane, Australia

5. Intensive Care Unit, Princess Alexandra Hospital, Brisbane, Australia

6. School of Health Sciences, City, University of London, United Kingdom

7. School of Medical Science, Griffith University, Gold Coast, Australia

8. Melanoma Institute Australia, Research and Biostatistics group

This work was performed at the Austin Hospital, Melbourne, Victoria, Australia

A/Prof Fealy, Prof Aitken, Dr du Toit, Dr Lo and Prof Baldwin have disclosed that they do not have any potential conflicts of interest.

\section{Corresponding author}

A.Prof. Nigel Fealy

Department of Intensive Care

Austin Hospital

Studley Rd, Heidelberg, Victoria 3084, Australia.

Tel: 61-3-9496 8403; Fax: 61-3-9496 3932E-mail: nigel.fealy@austin.org.au 


\section{Abstract}

Objective: To determine whether blood flow rate influences circuit life in continuous renal replacement therapy.

Design: Prospective randomized controlled trial.

Setting: Single centre tertiary level intensive care unit.

Patients: Critically ill adults requiring continuous renal replacement therapy.

Interventions: Patients were randomized to receive one of two blood flow rates: $150 \mathrm{~mL} / \mathrm{min}$ or $250 \mathrm{~mL} / \mathrm{min}$.

Measurements and Main Results: The primary outcome was circuit life measured in hours. Circuit and patient data were collected until each circuit clotted or was ceased electively for non-clotting reasons. Data for clotted circuits are presented as median (Inter-quartile range) and compared using the Mann Whitney $U$ test. Survival probability for clotted circuits was compared using log-rank test. Circuit clotting data were analysed for repeated events using hazards ratio (HR). One hundred patients were randomised with 96 completing the study (150 $\mathrm{mL} / \mathrm{min}, \mathrm{n}=49 ; 250 \mathrm{~mL} / \mathrm{min}, \mathrm{n}=47$ ) using 462 circuits (245 run at $150 \mathrm{~mL} / \mathrm{min}$ and 217 run at $250 \mathrm{~mL} / \mathrm{min})$. Median circuit life for $1^{\text {st }}$ circuit (clotted) was similar for both groups $(150 \mathrm{~mL} / \mathrm{min}$ : $9.1[5.5,26]$ hrs vs. $10[4.2,17] \mathrm{hrs} ; \mathrm{p}=0.37)$. CRRT using blood flow rate set at $250 \mathrm{~mL} / \mathrm{min}$ was not more likely to cause clotting compared to $150 \mathrm{~mL} / \mathrm{min}$ (HR, $1.00[0.60,1.69] ; p=0.68)$. Gender, Body Mass Index, weight, vascular access type, length, site, mode of CRRT or International Normalised Ratio had no effect on clotting risk. CRRT without anticoagulation was more likely to cause clotting compared to use of heparin strategies (HR 1.62, $p=0.003$ ). Longer Activated Partial Thromboplastin Time (HR 0.98, $p=0.002$ ] and decreased platelet count (HR $1.19 p=0.03]$ was associated with a reduced likelihood of circuit clotting.

Conclusions: There was no difference in circuit life whether using blood flow rates of 250 $\mathrm{mL} / \mathrm{min}$ or $150 \mathrm{~mL} / \mathrm{min}$ during CRRT. 
Key words: Continuous Renal Replacement Therapy, filter life, circuit life, continuous hemofiltration, acute kidney injury, critical care 


\section{Introduction}

Acute kidney injury (AKI) is a complication of critical illness that affects up to $50 \%$ of intensive care patients. ${ }^{1-3}$ The use of Renal Replacement Therapy (RRT) has evolved as the treatment for severe AKI and is required in $5-6 \%$ of all critically ill patients in intensive care units (ICUs). ${ }^{4}$ Continuous Renal Replacement Therapies (CRRT) relies on the maintenance of extracorporeal circuit (EC) patency for as long as possible, however premature circuit failure due to clotting may cause blood loss, reduced therapeutic efficacy and increased workload and treatment costs. ${ }^{5-8}$

Clogging and clotting of the hemofilter membrane is the major mechanism of premature failure and circuit loss in CRRT. ${ }^{9,10}$ It has been suggested that increasing blood flow rate (BFR) through the EC to speeds greater than $200 \mathrm{~mL} / \mathrm{min}$ may reduce premature clotting. ${ }^{11}$ One recent report demonstrated a reduction in filter lifespan when BFR was less than $200 \mathrm{~mL} / \mathrm{min}$ concluding that the optimal blood flow rate during CRRT is between 250$300 \mathrm{~mL} / \mathrm{min}^{12}$

The impact of BFR on membrane clotting rate is potentially important and has not been examined in controlled studies. Despite suggestions to increase blood flow rates in the $E C^{11,12}$, there remains great variability in the prescription of this setting worldwide. Although a recent survey of Australian and New Zealand ICUs indicated a BFR of $150-200 \mathrm{~mL} / \mathrm{min}$ was the dominant setting, a faster rate of $200-250 \mathrm{~mL} / \mathrm{min}$ was also commonplace in ICU's surveyed..$^{13}$ Observational studies and recent worldwide practice surveys of CRRT also demonstrate great variability in practice from $80 \mathrm{~mL} / \mathrm{min}^{12}$ to $350 \mathrm{~mL} / \mathrm{min} .{ }^{14-16}$ While BFR may 
be an important determinant of circuit life in CRRT, the most suitable speed to reduce clotting and optimise membrane life has not been identified. To address this question, we conducted a prospective randomized controlled trial (RCT). We tested the hypothesis that a faster blood flow rate $(250 \mathrm{~mL} / \mathrm{min})$ would be superior to a slower blood flow rate $(150 \mathrm{~mL} / \mathrm{min})$ in maintaining circuit patency in CRRT.

\section{Materials and Methods}

\section{Trial design and setting}

This study was a prospective, parallel group RCT conducted in a 24 bed, adult, tertiary intensive care in Melbourne, Victoria, Australia. The study was registered at the Australian New Zealand Clinical Trials Registry (ACTRN:12615001353583) and approved by Austin Health Human Research Ethics Committee (HREC project No. H2012/04772). Written informed consent was obtained from the patient or their next of kin prior to, or soon after enrolment.

\section{Eligibility criteria}

Critically ill patients in ICU were eligible for the study if they fulfilled three criteria: 1) age $\geq 18$ yrs, 2) AKI (RIFLE classification $F)^{16}$ requiring CRRT and 3) vascular access was via the femoral vein for standardisation. Patients were considered ineligible for the study if they fulfilled any of the following exclusion criterion: 1 ) required citrate anticoagulation (citrate protocol requires set blood flow rate of $150 \mathrm{~mL} / \mathrm{min}), 2$ ) expected stay in the ICU was less than 24 hours. 


\section{Interventions}

The study compared two blood flow rate settings on circuit life in CRRT. The intervention was a set BFR of $250 \mathrm{~mL} / \mathrm{min}$ and the control was a set BFR of $150 \mathrm{~mL} / \mathrm{min}$. CRRT was performed using Continuous VenoVenous Hemofiltration (CVVH) or Continuous VenoVenous Hemodiafiltration (CVVHDF) modalities. Vascular access was via either Niagara 13.5 Fr-catheter $(24 \mathrm{~cm})$ (Bard, Murray Hill, NJ, USA) or Gamcath Dolphin Protect $13.0 \mathrm{Fr}$ catheter $(25 \mathrm{~cm})$ (Gambro, Hechingen, Germany) dual lumen catheters. Treatment modality and choice of vascular access was at the discretion of the treating physician. Prismaflex with AN69ST (ST100) $1.0 \mathrm{~m}^{2}$ membrane (Gambro Nephral TM, Lund, Sweden) or Infomed HF 440 with DF 140 Polyethersulfone $1.4 \mathrm{~m}^{2}$ membrane (Infomed, Geneva, Switzerland) was used for all treatments. Bicarbonate buffered replacement and dialysis fluid (Baxter, Castlebar, Co. Mayo, Ireland) was used. In CVVH, replacement fluid was delivered into the EC before and after the filter (pre and post-dilution), with a ratio of $50 \%$ predilution and $50 \%$ postdilution. Dose in CVVH was standardised at $2000 \mathrm{~mL} /$ hour. In CVVHDF, the replacement fluid was all delivered postdilution. Dose in CVVHDF was standardised at $1000 \mathrm{~mL} /$ hour replacement and $1000 \mathrm{~mL} /$ hour dialysate.

Anticoagulation was provided according to a predefined ICU protocol and mandates no anticoagulation in patients at risk of bleeding from a coagulopathy or thrombocytopaenia. Options for anticoagulation when used included regional heparinisation with unfractionated heparin (1000 IU/hour) delivered prefilter and protamine (10 $\mathrm{mgs} /$ hour) delivered in the return limb of the EC for reversal of heparin. Unfractionated heparin was used alone and 
delivered pre-filter at 5-10 IU/kg/hour. CRRT was prescribed by the treating intensivist and delivered by ICU nurses. The decision to start or stop CRRT, and determining the reason for stopping, was done by ICU doctors and nurses respectively as is usual protocol for the ICU.

\section{Data collection}

Baseline data relating to age, gender, weight, BMI, source of admission, severity of illness (Acute Physiology and Chronic Health Evaluation score II, III; Simplified Acute Physiology Score II), diagnostic group, presence of sepsis, mechanical ventilation, inotropes/vasopressors, and basic laboratory variables pertaining to renal function was collected.

\section{Outcomes}

The primary outcome was circuit life (measured in hours) and recorded as clotted when; 1) Transmembrane pressure across the circuit exceeded $300 \mathrm{mmHg}$, 2) pre-filter pressure $>200 \mathrm{mmHg}, 3$ ) visible clot obstructing flow through the circuit and 4) the blood pump was unable to rotate due to clot obstruction in the membrane or for 'elective' reasons. e.g. CT, MRI, surgical intervention or cessation prior to clotting for native renal assessment.

\section{Sample size}

Without supportive data to inform a power calculation for this study, 100 patients were chosen to ensure a sample that was sufficient to reflect usual ICU patient characteristics and allow recruitment completion in one year. Patients stayed in the BFR treatment group allocated at randomization. The treating physician prescribed the CRRT modality for each 
patient (CVVH or CVVHDF) which was maintained/retained for all subsequent treatments. All circuits used for these patients were included and analysed accordingly.

\section{Randomization}

Patients were screened and entered the study by ICU clinical staff. Patients were randomly assigned with stratification for mode (CVVH or CVVHDF). Once the treating physician prescribed CRRT and the mode of therapy, patients were randomized using a web based central randomization service. A variable block randomization with parallel allocation was used to allocate to each study group (150mL/min vs. $250 \mathrm{~mL} / \mathrm{min})$.

\section{Statistical methods}

The primary outcome (circuit life) was analysed in a two-step process. First analysis: this excluded all electively removed or non-clotted circuits from the data. The distribution of data for all circuits meeting the defined clotting criteria was then assessed. As expected study variables were not normally distributed and non-parametric statistics were used with circuit life reported as median and IQR. Circuit life for the two groups (150 mL/min vs. $250 \mathrm{~mL} / \mathrm{min}$ ) was compared using Mann-Whitney U-test. Analysis of the two groups was then assessed for survival probability and presented graphically using Kaplan-Meier survival plots. A log-rank test was used to compare circuit life between the two groups. This analysis was not adjusted for any confounding variables.

Second analysis: included all circuits (clotted and those electively removed). Circuit life was analysed using repeated events survival analysis. ${ }^{7,17}$ A proportional hazard conditional 
frailty model ${ }^{17}$ (an extension of cox regression) was used to test for within patient dependence. It was expected that there would be heterogeneity among individual trial patients. In addition, individual trial patients may contribute one or multiple circuits. It was assumed to be a correlation between an individual trial patient contributing multiple circuits and circuit life. The frailty model was used to test event dependence (where the event is a clotted circuit) within the trial patients. Event dependence in this study meant that an occurrence of one event (time to clotting of the circuit) may make further events (additional circuit clotting times) more or less likely. The advantage of this model is that it considers any within-cluster correlation of circuit life. Proportions were compared using a chi-square test.

First analysis was carried out using IMB SPSS statistics for Windows (v2, IBM Corp., Armonk, NY). Second analysis was performed using SAS (Enterprise Guide v5.1; SAS Institute Inc., Carey, NC).

\section{Results}

\section{Participants and recruitment}

One hundred patients were randomized between June 2013 and August, 2014. Two patients from each group were randomized but did not receive CRRT. The CONSORT diagram for patient enrolment is shown in Figure 1. Overall, 96 patients ( 49 in the $150 \mathrm{~mL} / \mathrm{min}$ group and 47 in the $250 \mathrm{~mL} / \mathrm{min}$ group) contributed a total of 463 study circuits: 245 in the 150 $\mathrm{mL} / \mathrm{min}$ group and 218 in the $250 \mathrm{~mL} / \mathrm{min}$ group. Median study circuits per patient was four (IQR 2, 6) and totalled 8206 CRRT treatment hours. 
At randomization, patients were similar with respect of age, sex, severity of illness scores (APACHE II, III, SAPS II), admission source and diagnosis (Table 1.). There was a nine kg difference in patient weight between the two groups $(p=0.03)$; however, BMI was similar for both groups. Pre-randomization renal laboratory variables were also similar for both groups.

\section{Primary outcomes - Circuit Life}

The first analysis incorporated 369 defined clotted circuits. The median circuit life for these circuits ( $n=369)$ was similar for both groups $(150 \mathrm{~mL} / \mathrm{min}, \mathrm{n}=196$ [10 hrs IQR 6.0, 24]) vs. $(250 \mathrm{~mL} / \mathrm{min}, \mathrm{n}=173$ [11.5 hrs IQR 6.8, 18.3] $\mathrm{p}=0.81)$. For first analysis, there were 81 clotted first circuits. The median circuit life of these circuits was $9.1 \mathrm{hrs}$ (IQR, 5.5, $26 \mathrm{hr}$ ) in the $150 \mathrm{~mL} / \mathrm{min}$ group compared to $10 \mathrm{hrs}$ (IQR, 4.2, $17 \mathrm{hr}$ ) in the $250 \mathrm{~mL} / \mathrm{min}$ group, $\mathrm{p}=0.37$. Second and third median circuit lives for those deemed to have clotted were also similar (Table 2). The probability of the first study circuit from each patient failing due to clotting did not differ between BFR groups $(150 \mathrm{ml} / \mathrm{min}$ vs. $250 \mathrm{~mL} / \mathrm{min} ; \mathrm{HR}, 0.85$, log rank test $=0.46)$ (Figure 2).

The second analysis involved evaluation of all circuit terminations (clotted and electively removed) and revealed that a BFR of $250 \mathrm{~mL} / \mathrm{min}$ was not more likely to cause clotting compared to $150 \mathrm{~mL} / \mathrm{min}(\mathrm{HR}, 1.00$ [0.60, 1.69]; $p=0.68$, variance of the random effect, 1.078 [0.23]) (Table 3). There were no differences in likelihood of clotting for: gender, BMI, weight, vascular access type, length or site, mode of CRRT or INR. CRRT without use of anticoagulation was more likely to cause clotting compared to use of heparin or heparin/protamine (HR 1.62, [1.18, 2.23], $\mathrm{p}=0.003)$. Longer APTT (HR 0.98, [0.97, 0.99], 
$p=0.002$ ) was associated with a lower likelihood of circuit clotting. Probability of clotting was higher in those patients with higher platelet counts (HR 1.19, [1.01, 1.40], $p=0.03$ ) (Table 3).

\section{Discussion}

This is the first known prospective study to examine the effect of blood flow rate on circuit duration in both CVVH and CVVHDF. In a cohort of 100 ICU patients requiring CRRT three key findings have been identified: first, when blood flow rate is increased to $250 \mathrm{~mL} / \mathrm{min}$ it does not increase circuit life during CRRT. Second, the use of an anticoagulation strategy and longer APTT's extends CRRT circuit life. Third, patients with higher platelet counts were more prone to premature circuit clotting in this study.

\section{Relationship to previous studies}

The maintenance of circuit patency by prevention of clotting is the greatest challenge associated with providing CRRT for critically ill patients. As a result, many studies have focused on anticoagulation strategies to extend circuit life, ${ }^{7,18-27}$ while many aspects of treatment and prescription setting have not been investigated. One RCT has included blood flow rate in the assessment of circuit clotting in CRRT, indicating that blood flow rates $>125 \mathrm{~mL} / \mathrm{min}$ did not improve circuit survival. ${ }^{28}$ This study was conducted in CVVHD mode which is rarely used in current practice. ${ }^{13,15,29,30}$ Pure diffusive modes of hemofiltration such as CVVHD have been shown to be associated with decreased procoagulatory activity in the dialyser membrane when compared to convective modes ${ }^{31,32}$ and makes comparisons to $\mathrm{CVVH}$ and CVVHDF problematic. 
One single centre study assessing 1332 treatments from 355 patients concluded that BFR did indeed affect circuit life. ${ }^{12}$ In this retrospective audit, the authors suggest a BFR less than $200 \mathrm{~mL} / \mathrm{min}$ significantly decreased circuit life compared to rates greater than 200 $\mathrm{mL} / \mathrm{min}$. They also determined that BFR greater than $300 \mathrm{~mL} / \mathrm{min}$ led to lower median circuit lives and recommended an optimal BFR of between $250-300 \mathrm{~mL} / \mathrm{min}$.

\section{Implications of study findings}

Our data provides evidence to suggest that a faster blood flow rate does not influence circuit life and prescription of rates greater than $150 \mathrm{~mL} / \mathrm{min}$ makes no difference to the likelihood of clotting in either CVVH or CVVHDF. It has previously been suggested that blood flow should be maintained at $200 \mathrm{~mL} / \mathrm{min}^{33-35}$ and always be greater than $100 \mathrm{~mL} / \mathrm{min}$ to avoid premature clotting. ${ }^{36}$ The ability to maintain consistent and constant flow may be more critical, with the flow and resistance balance being more important. We have previously reported these mechanical factors and their adverse effect on circuit life. ${ }^{10,37,38}$

Despite many authors suggesting blood flow rates for CRRT of $200-250 \mathrm{~mL} / \mathrm{min}^{12,33-35}$ and international surveys indicating practical prescriptions of $>200 \mathrm{~mL} / \mathrm{min}$; there has been no endorsements for this setting. The Acute Dialysis Quality Initiative (ADQI) consensus guidelines for operational characteristics from 2002 indicate that blood flow may be increased to augment solute clearance but do not include a recommendation for this prescription. ${ }^{39}$ The more recent Kidney Disease Improving Global Outcomes (KDIGO) consensus guidelines outline settings for different RRT modalities indicating $150-250 \mathrm{~mL} / \mathrm{min}$ is typically prescribed for CRRT modes such as CVVH and CVVHDF but make no recommendations for practice based 
on evidence. ${ }^{40}$

The use of anticoagulants to prevent extracorporeal clotting and extend circuit life in CRRT has been used for decades. ${ }^{41}$ Unfractionated heparin (UFH) remains the most commonly prescribed anticoagulant ${ }^{13,42}$ worldwide and remains the standard against which other anticoagulant regimens are compared. ${ }^{7,21,22,24,25,43}$. A regional heparin technique favours patient safety and anticoagulant free CRRT is used for a high risk of bleeding. ${ }^{44,45}$

We have previously reported on circuit life in CVVH when no anticoagulation was used compared to low dose UFH and a regional heparin technique. ${ }^{45}$ This study of 300 filters described similar circuit lives for all three methods and has similarities to our findings which indicate the strong association between higher platelet counts and premature circuit clotting.

\section{Strengths and Limitations}

This RCT of 100 patients presents for the first time the outcomes of an investigation into the effect of blood flow rate on circuit life in two commonly used modes of CRRT. This analysis is based on a large number of circuits and for 8206 hours of treatment time. This number of patients and treatment time is representative of a tertiary level intensive care unit and signifies important findings for current CRRT practice. The presentation of our analysis for first circuit (clotted) and the analysis of all circuits using repeated events survival analysis should be the new standard for studies reporting circuit life in CRRT where previously an all circuits analysis may have drawn conclusions not valid due to repeated measures effect. The study was not powered to detect a difference as there was insufficient historical data available 
to make the appropriate group size calculation. One further limitation may be the defined blood flow rates used in this study. Blood flow rates less than $150 \mathrm{~mL} / \mathrm{min}$ or greater than 250 $\mathrm{mL} / \mathrm{min}$ may have yielded a different outcome. Circuit life (hrs) in both groups may be shorter due to the large proportion of patients enrolled have a diagnosis of liver failure and liver transplantation making comparisons to other ICU's difficult. Two membrane compositions were used and anticoagulation according to an established local policy. These two factors may have some influence on our findings.

\section{Conclusions}

A blood flow rate of $250 \mathrm{~mL} / \mathrm{min}$ does not improve CRRT circuit life compared with a blood flow rate of $150 \mathrm{~mL} / \mathrm{min}$. Independent factors that may extend circuit life include anticoagulation strategies, higher APTT and lower platelet counts.

\section{Acknowledgements}

We thank the medical and nursing staff of the Austin intensive care unit for their support of this study.

Figure 1. Flow diagram of participants showing assessment of eligibility, enrollment, treatment allocation and followup in the trial. CRRT = Continuous Renal Replacement Therapy, CVVH = Continuous VenoVenous Hemofiltration, CVVHDF = Continuous VenoVenous Hemodiafiltration

Figure 2. Kaplan Meier estimate of the probability of continuous renal replacement therapy circuit survival for the first circuit - clotted circuits only 


\section{References}

1. Hoste EAJ, Bagshaw SM, Bellomo R, et al: Epidemiology of acute kidney injury in critically ill patients: the multinational AKI-EPI study. Intensive Care Med 2015; 41:1411-1423

2. Ostermann M, Chang RW: Acute kidney injury in the intensive care unit according to RIFLE. Crit Care Med 2007; 35: 1837-1843

3. Uchino S, Kellum J, Bellomo R et al: Acute renal failure in critically ill patients: a multinational multicentre study. JAMA 2005; 294: 813-818

4. Dennen P, Douglas IS, Anderson R: Acute Kidney Injury in the intensive care unit: an update and primer for the intensivist. Crit Care Med 2010; 38: 261-275

5. Bellomo R, Mårtensson J, Lo S, et al: Femoral access and delivery of continuous renal replacement therapy dose. Blood Purif 2016; 41:11-17

6. Fealy N, Baldwin I, Bellomo R: The effect of circuit "down-time" on uraemic control during continuous veno-venous hemofiltration. Crit Care Resusc 2002; 4:266-270

7. Gattas DJ, Rajbhandari D, Bradford C, et al: A randomized controlled trial of regional citrate versus regional heparin anticoagulation for continuous renal replacement therapy in critically ill adults. Crit Care Med 2015; 43(8):1622-1629

8. The RENAL Replacement Therapy Study Investigators, Bellomo R, Cass A, Cole L, et al: Intensity of continuous renal replacement therapy in critically ill patients N Engl J Med 2009; 361: $1627-38$ 
9. Ward D. Principles of extracorporeal circulation. In Ronco C, Bellomo R, Kellum JA. editors. Critical Care Nephrology, $2^{\text {nd }}$ ed. Philadelphia Elsevier Saunders 2009; p. 1141-1145

10. Baldwin I, Bellomo R, Koch B: Blood flow reductions during continuous renal replacement therapy and circuit life. Intensive Care Med 2004; 30:2074-2079

11. Ronco C, Ricci Z, De Backer D, et al: Renal Replacement therapy in acute kidney injury: controversy and consensus. Crit Care 2015; 19:146

12. Dunn WJ, Sriram S: Filter lifespan in critically ill adults receiving continuous renal replacement therapy: the effect of patient and treatment related variables Crit Care Resusc 2014; 16(3): 225-231

13. Fealy N, Aitken L, du Toit E, et al: Continuous renal replacement therapy: current practice in Australian and New Zealand intensive care units. Crit Care Resusc 2015; 17:83-91

14. Uchino S, Toki N, Ohnuma $\mathrm{T}$, et al: Validity of low intensity continuous renal replacement therapy. Crit Care Med 2013; 41(11):2584-2591

15. Jones S, Devonald MAJ: How acute kidney injury is investigated and managed in UK intensive care units - a survey of current practice. Nephrol Dial Transplant 2013; 28: 11861190

16. Bellomo R, Ronco C, Kellum JA, et al: Acute renal failure - definition, outcome measures, animal models, fluid therapy and information technology needs: the Second International Consensus Conference of the Acute Dialysis Quality Initiative (ADQI) Group. Crit Care 2004; 
17. Box-Steffensmeier JM, De Bouf S: Repeated events survival models: The conditional frailty model. Stat Med 2004; 25:3518-3533

18. Oudemans-van Straaten HM, Bosman RJ, Koopmans M et al: Citrate anticoagulation for continuous veno-venous hemofiltration. Crit Care Med 2009; 37:545-552

19. Tolwani A, Wille KM: Advances in continuous renal replacement therapy: citrate anticoagulation update Blood Purif 2012; 34(2): 88-93

20. Oudemans-van Straaten HM, Wester JP, de Pont AC, et al: Anticoagulation strategies in continuous renal replacement therapy: can the choice be evidence based? Intensive Care Med $2006 ; 188-202$

21. Bai M, Zhou M, He L et al: Citrate versus heparin anticoagulation for continuous renal replacement therapy: an updated meta-analysis of RCTs. Intensive Care Med 2015; 41:20982110

22. Monchi M, Berghmans D, Ledoux D, et al: Citrate vs. heparin for anticoagulation in continuous venovenous hemofiltration: a prospective randomized study. Intensive Care Med $2004 ; 30(2): 260-265$

23. van de Wetering J, Westendorp RG, van der Hoeven JG, et al: Heparin use in continuous renal replacement procedures: the struggle between circuit coagulation and patient haemorrhage. J Am Society Neph 1996; 7:145-150 
24. Joannidis $M$, Kountchev J, Rauchenzauner $M$ et al: Enoxaparin versus unfractionated heparin for anticoagulation during continuous veno-venous hemofiltration - a randomized controlled crossover study. Intensive Care Med 2007; 33: 1571-1579

25. Schilder L, Nurmohamed SA, Bosch FH et al. CASH study group: Citrate anticoagulation versus systemic heparinisation in continuous venovenous hemofiltration in critically ill patients with acute kidney injury: a multi-center randomized clinical trial. Crit Care 2014; 18(4): 472

26. Lee YK, Lee HW, Choi KH, et al: Ability of nafamostat mesilate to prolong filter patency during continuous renal replacement therapy in patients of high risk of bleeding: A randomized controlled study. PLos One 2014; 9(10): e108737

27. Gainza FJ, Quintanilla N, Pjoan Jl, et al: Role of prostacyclin (epoprostenol) as an anticoagulant in continuous renal replacement therapies: efficacy, security and cost analysis. J Nephrol 2006; 19(5): 648-655

28. Ramesh Prasad GV, Palevsky P, Burr R, et al: Factors affecting system clotting in continuous renal replacement therapy: results of a randomized controlled trial. Clin Nephrol 1998; 53(1): $55-60$

29. Legrand M, Darmon M, Joannidis M, et al: Management of renal replacement therapy in ICU patients: an international survey Intensive Care Med 2013; 39: 101-108

30. Jamal JA, Mat-Nor MB, Mohamad-Nor FS, et al: A national survey of renal replacement therapy prescribing practice for acute kidney injury in Malaysian intensive care units. Nephrol 
$2014 ; 19: 507-512$

31. Klingel $R$, Schaefer $M$, Schwarting A, et al: Comparative analysis of procoagulatory activity of haemodialysis, hemofiltration and haemodiafiltration with a polysulfonemembrane (APS) and with different modes of enoxaparin anticoagulation. Nephrol Dial Transplant 2004; 19(1): $164-170$

32. Ricci Z, Ronco C, Bachetoni A, et al: Solute removal during continuous renal replacement therapy in critically ill patients: convection versus diffusion. Crit Care 2006; 10:R67

33. Baldwin I: Factors affecting circuit patency and circuit life. Contrib Neph 2007; 156: 178184

34. Baldwin I, Bridge N, Elderkin T: Nursing issues, practices and perspectives for the management of continuous renal replacement therapy in the intensive care unit. In: Ronco C \& Bellomo R (Eds). Critical Care Nephrology ( $1^{\text {st }}$ Ed). Dordecht: Klower Academic Publishers 1998; p. 1309-1325

35. Davies H, Leslie G: Maintaining the CRRT circuit: non-anticoagulant alternatives. Australian Crit Care 2006; 19(4): 133-138

36. Kox WJ, Rohr U, Waurer H: Practical aspects of renal replacement therapy. Int J Artif Organs 1996; 19: 100-105

37. Baldwin I, Fealy N, Carty $\mathrm{P}$, et al: Bubble chamber clotting during continuous renal replacement therapy: vertical versus horizontal blood flow entry. Blood Purif 2012; 34:213- 
38. Inbyung K, Fealy N, Baldwin I, et al: Premature circuit clotting due to likely mechanical failure during continuous renal replacement therapy. Blood Purif 2010; 30: 79-83

39. Palevsky P, Bunchman T, Tetta C: The Acute Dialysis Quality Initiative - part V: Operational characteristics of CRRT. Adv Ren Replace Ther 2002; (9)4: 268-272

40. Kidney Disease: Improving Global Outcomes (KDIGO) Acute Kidney Injury Work Group. KDIGO Clinical Practice Guideline for Acute Kidney Injury. Kid Int Supp 2012; 2: 1-138

41. George CRP: Hirudin, heparin and Heinrich Necheles. Nephrol 1998; 4: 225-228

42. Uchino $\mathrm{S}$, Bellomo $\mathrm{R}$, Morimatsu $\mathrm{H}$ et al: Continuous renal replacement therapy: $a$ worldwide practice survey. The beginning and ending supportive therapy for the kidney (BEST kidney) investigators. Intensive Care Med 2007; 33: 1563-1570

43. Mei-Yi W, Yung-Ho H, Chyi-Huey B, et al: Regional citrate versus heparin anticoagulation for continuous renal replacement therapy: A meta-analysis of randomized controlled trials. Am J Kidney Dis 2012; 59(6): 810-818

44. Nongnuch A, Tangsujaritvijit V, Davenport A: Anticoagulation for renal replacement therapy for patients with acute kidney injury. Minerv Urol Neph 2016; 68: 87-104

45. Uchino S, Fealy N, Baldwin I, et al: Continuous venovenous hemofiltration without anticoagulation. ASAIO 2004; 50(1): 76-80 